\title{
The Current Status of Knowledge and Behavior Related to STI Prevention among High School Students in Korea
}

\author{
Jungok Yu ${ }^{1}$, Yuko Tanaka, ${ }^{2, *}$ Yuko Yamaguchi ${ }^{3}$, Hiroya Matsuo ${ }^{4}$ \\ ${ }^{1}$ Department of Nursing, Dong-A University, Busan, Korea \\ ${ }^{2}$ Department of School Health Sciences, Tokushima University Graduate School, Tokushima, Japan \\ ${ }^{3}$ Department of Gerontological Nursing, Kobe University Graduate School of Health Sciences, Kobe, Japan \\ ${ }^{4}$ Department of Public health, Kobe University Graduate School of Health Sciences, Kobe, Japan
}

Copyright $\bigcirc 2018$ by authors, all rights reserved. Authors agree that this article remains permanently open access under the terms of the Creative Commons Attribution License 4.0 International License

\begin{abstract}
This study explores coping with STIs and related factors including knowledge, sexual behavior, and sex education among Korean adolescents. Anonymous self-administered questionnaires were distributed to 135 male and 150 female high school students. The results showed that $2.1 \%$ of participants had had sexual experience. Males had less information than females $(\mathrm{p}<0.05) ; 38.5 \%$ of males did not consult anyone. In contrast, females acquired information from and consulted with their parents. Although females' sexual abstinence behavior was higher than that of males $(p<0.001)$, there were no significant differences in the STI knowledge test. Regarding sex education, $32.4-59.5 \%$ of students had received education on STIs. Students' degree of satisfaction with sexual knowledge was $10.2 \%$, their understanding of STIs was poor, and they could not cope with having a checkup. In conclusion, high school students receive insufficient education to protect themselves from STIs. Their knowledge was not related to sexual abstinence behavior and coping behavior. Therefore, schools in Korea should provide high school students with appropriate education on STI prevention and coping behavior. In addition, an environment that facilitates consultation and clinics for helping young people deal with sexual problems should be provided.
\end{abstract}

Keywords Education on STI Prevention, High School Students, Korea

\section{Introduction}

More than one million people worldwide contract sexually transmitted infections (STIs) every day [1]. STIs affect people of all age groups, but it has been reported in the U.S. that half of patients who contract STIs are 15- to 24-year-old adolescents [2] and that half of women past puberty with sexual experience contract STIs such as chlamydia or human papillomavirus [3]. In Korea, 1 out of $14(7.3 \%)$ adolescents have experienced STIs [4] and the number of adolescents aged 15 to 19 receiving STI treatment has been increasing, from 8,511 in 2013 to 10,571 in 2016 [5].

The age of Korean adolescents at first sexual intercourse has continued to decrease, with the average age being reported as 13.1 years old [6]. STIs do not usually have symptoms; chlamydia, which is a frequent infection in young people, is asymptomatic. Further, adolescents do not receive checkups even when they have symptoms, so the number of STI patients is regarded as higher than reported. Thus, it is necessary to pay more attention to STI prevention and care for adolescents. Some STIs, if left untreated, may lead to serious complications, such as inflammatory disease in the pelvis, sterility, and ectopic pregnancy [7]. Given that STIs during adolescence can have substantial impacts on the health of genital organs in adulthood, it is important for adolescents to prevent them and treat them early.

According to a recent study, the most common STIs among adolescents are chlamydia followed by gonorrhea [8]. Further, adolescents usually avoid examination and miss the treatment window because most of these STIs have no symptoms or have only minor symptoms [9]. Previous studies have shown that early examination is not carried out well because adolescents lack knowledge of the characteristics and spread of STIs and do not take STIs seriously [10-12]. Moreover, concern about being stigmatized, lack of money to see a doctor, and fear of examination methods have been identified as factors hindering the early discovery and treatment of STIs [10-12].

While $72 \%$ of Korean adolescents reported having received sex education in the school classroom or through the media in the past year [6], only $2.7 \%$ had received it once a week, $8.7 \%$ once a month, and $90 \%$ about $1-2$ times 
a semester or just once a year [13]. In addition, the sex education that students received at school mainly focused on sexual violence, dating, and puberty changes. In contrast, students wanted to learn about contraception, AIDS, and sexually transmitted diseases [14]. Thus, there is a difference between the content of sex education taught at school and the learning interests of students. However, there is a lack of research on STIs among adolescents in Korea; only the prevalence of STIs and some risk factors have been studied $[4,15,16]$.

Therefore, in order to enhance STI prevention education programs for adolescents, this study examines how much Korean adolescents know about STI prevention and how they practice sexual abstinence behavior and cope with STI symptoms.

\section{Materials and Methods}

\subsection{Design}

This was a cross-sectional study conducted to identify the status of STI prevention-related knowledge and coping methods among high school students in Korea.

\subsection{Material}

\subsubsection{Participants and Data Collection}

The study covered four types of public high school in Busan, Korea in June 2017. Participants were recruited from two classes $(n=35-40)$ from one boys' high school (25 classes), one girls' high school (30 classes) and one general (coeducational) high school (24 classes). In the vocational high school (coeducation), the number of students in classes was small $(\mathrm{n}=20-25)$, so we selected three classes from the 33 classes that had the same number of participants as those of the other types of school. The required sample size was calculated using OpenEpi software version 3.03a. According to another study [17] conducted by a co-researcher of the present study using the same variables, $19 \%$ of Thai adolescents had little knowledge of STI prevention and $11 \%$ coped inappropriately with STIs, with a 95\% confidence interval, so we required 151-237 students for this study. As a result, we selected 297 students from four schools as the required sample size, assuming that $20 \%$ of responses to the survey questionnaire would be inappropriate or unqualified. Finally, data from 285 participants were analyzed.

To recruit research participants, we put up an announcement on the bulletin board in the hallway outside the infirmaries of the sample high schools, and recruited students wishing to participate in the study. The purpose and method of the study were explained to the principal, teachers and students. The students were asked to explain the study to their parents or guardians at home using a written explanation. The consent of the parents or guardians and students was obtained. The self-administered survey questionnaire was distributed only to those students who gave their consent to the researcher, and completed questionnaires were collected using a secure box. The survey took about 10 to 15 minutes to complete.

\subsection{Method}

\subsubsection{Questionnaire}

This study used a self-administered questionnaire that consisted of the following questions.

General characteristics, sex behavior, and sex education. A self-administered questionnaire was distributed to the participants, inquiring about their personal information (gender, age), sources of information on sex-related knowledge, experience of sexual intercourse, counselors for discussing sexual problems and satisfaction with sexual knowledge. Questions on sex education included when they received sex education, who gave them sex education, and what they learned from the sex education.

\subsubsection{Knowledge on STI Prevention.}

This study used the STI test of Yamaguchi et al. [17]. A Korean researcher translated the original version according to the backward translation technique [18]. Knowledge of STI prevention was assessed with 12 "Yes/No" questions; 1 point was given for a correct response and 0 points for an incorrect response, resulting in a total score ranging from 0 to 12 . The higher the score, the higher the STI-related knowledge level.

\subsubsection{Sexual Abstinence Behavior}

The Sexual Abstinence Behavior scale of Norris et al. was used [19]. This scale consists of four items: decision-making ("Tell yourself you were making the right decision by waiting to have sex."), interacting ("Say 'No' to sex."), negotiation ("Tell her/him that you want to wait to have sex."), and peer pressure ("Avoid being pressured into having sex by making sure you are out with a group of people."). A 6-point Likert scale was used, from $1=$ strongly disagree to $6=$ strongly agree. Cronbach's alpha was .80 for the total. The total score was 1-24. A higher score means stronger sexual abstinence behavior.

\subsubsection{Coping Behavior for STIs.}

To identify adolescents' coping behavior for STI symptoms, a tool created by Yamaguchi et al. [17] was used to present case examples of chlamydia (mild symptoms) and gonorrhea (strong symptoms) for males and females and to ask how they would cope with each set of symptoms. Responses on coping with STIs were categorized by attending or not attending a medical checkup; responses of the latter were followed by additional questions about the reasons and other methods of coping with STI symptoms. This tool was also translated according to the backward translation technique 
[18].

\subsection{Data analysis}

The collected data were analyzed using SPSS Statistics ver. 23.0. The participants' general characteristics and sex education characteristics were represented by frequencies and percentages, and the STI knowledge level of participants was summarized using means and standard deviations. Medical checkups for the symptoms of chlamydia and gonorrhea, as well as reasons for not receiving checkups and alternative coping methods, were represented by frequencies and percentages. We analyzed gender differences with the Chi-square test, Mann-Whitney U test and then used the Chi-square test to identify the relation between STI prevention education and satisfaction with sexual knowledge, STI knowledge test and medical checkup.

\subsection{Ethical considerations}

This study was approved by D University's Institutional Review Board (201701-HR-001-02). Principals, teachers in four schools, all participants and their parents or guardians provided written consent prior to answering the questionnaires.

\section{Results}

\subsection{General characteristics}

Table 1 lists the 285 high school students who participated in this study. Of the total, $47.4 \%$ were male students and $52.6 \%$ were female students. Mean age was 17.0 \pm 0.7 years old, and there was no significant difference among gender $(p=0.12)$. Most respondents were in their second year of high school, and $97.5 \%$ of students were living with their parents. Regarding sex, $2.1 \%$ of students had experience of sexual intercourse. There was no significant difference among gender.

Table 1. General characteristics

\begin{tabular}{|c|c|c|c|c|}
\hline General characteristics & & $\mathrm{n}$ & (\%) & $\mathrm{p}$ \\
\hline \multirow[t]{2}{*}{ Gender } & Male & 135 & $(47.4)$ & \\
\hline & Female & 150 & $(52.6)$ & \\
\hline \multirow[t]{3}{*}{ High school } & First year & 52 & $(18.2)$ & \\
\hline & Second year & 159 & $(55.8)$ & \\
\hline & Third year & 74 & $(26.0)$ & \\
\hline \multirow[t]{3}{*}{ Age : Me an \pm SD } & Total & & $17.0 \pm 0.7$ & \\
\hline & Male & & $17.1 \pm 0.7 \neg$ & \multirow{2}{*}{ n.s. } \\
\hline & Female & & $17.0 \pm 0.7$ & \\
\hline \multirow[t]{3}{*}{$\dagger$ Living with parents (Yes) } & Total & 278 & $(97.5)$ & \multirow{3}{*}{ n.s. } \\
\hline & Male & 132 & $(97.8)$ & \\
\hline & Female & 146 & $(97.3)$ & \\
\hline \multirow[t]{3}{*}{$\dagger$ Sexual intercource experiences } & Total & 6 & $(2.1)$ & \\
\hline & Male & 5 & $(3.7) \sqsupset$ & \multirow{2}{*}{ n.s. } \\
\hline & Female & 1 & (0.7) & \\
\hline
\end{tabular}

\subsection{Information Sources, Counselors for Sexual Problems, and Satisfaction with Sexual Knowledge}

The information sources of their current knowledge of sex (in order from highest to lowest) were school, internet/social networks [SNS], friends, media/TV, and home/parents (Table 2). Around $70 \%$ of students got sexual knowledge from school. Female students got more information from school and home/parents than male students $(p=.014$ and $p=.018)$. The main counselors for sexual problems (in order from highest to lowest) were parents, friends, and teachers, but nearly a third said they did not have anyone to consult. $45.3 \%$ of females consulted their parents, but only $17.8 \%$ of males did, showing a significant difference among gender $(p=.000)$. Furthermore, $38.5 \%$ of males and $22.7 \%$ of females had no counselor, showing a significant difference among gender $(\mathrm{p}=.004)$. 
Table 2. Information source on sex, the main counselor for sexual problems and Satisfaction with sexual Knowledge

\begin{tabular}{|c|c|c|c|c|c|c|c|}
\hline & \multicolumn{2}{|c|}{$\begin{array}{c}\text { Total } \\
(\mathrm{N}=285)\end{array}$} & \multicolumn{2}{|c|}{$\begin{array}{l}\text { Male } \\
(n=135)\end{array}$} & \multicolumn{2}{|c|}{$\begin{array}{l}\text { Female } \\
(n=150)\end{array}$} & \multirow[b]{2}{*}{$\mathrm{p}$} \\
\hline & $\mathrm{n}$ & $(\%)$ & $\mathrm{n}$ & $(\%)$ & $\mathrm{n}$ & (\%) & \\
\hline \multicolumn{8}{|c|}{ Information source (multiple answers) } \\
\hline 1. School & 209 & $(74.1)$ & 90 & $(67.2)$ & 119 & $(80.4)$ & .014 \\
\hline 2. Internet & 162 & (57.4) & 77 & (57.5) & 85 & (57.4) & n.s. \\
\hline 3. Friends & 114 & $(40.4)$ & 51 & $(38.1)$ & 63 & $(42.9)$ & n.s. \\
\hline 4. Media/TV & 91 & $(32.3)$ & 48 & $(35.8)$ & 43 & $(29.1)$ & n.s. \\
\hline 5. Home/Parents & 69 & $(24.5)$ & 24 & $(17.9)$ & 45 & $(30.4)$ & .018 \\
\hline 6. Hospital/Clinic & 19 & $(6.7)$ & 10 & $(7.5)$ & 9 & (6.1) & n.s. \\
\hline 7. Magazine/Comics & 12 & $(4.3)$ & 5 & (3.7) & 7 & (4.7) & n.s. \\
\hline$\dagger 8$. Partner & 9 & $(3.2)$ & 6 & $(4.5)$ & 3 & $(2.0)$ & n.s. \\
\hline$\dagger 9$. Others & 2 & $(0.7)$ & 0 & 0.0 & 2 & (1.4) & n.s. \\
\hline \multicolumn{8}{|l|}{ The best counselor } \\
\hline 1. Parent & 92 & $(32.3)$ & 24 & $(17.8)$ & 68 & $(45.3)$ & .000 \\
\hline 2. None & 86 & $(30.2)$ & 52 & $(38.5)$ & 34 & $(22.7)$ & .004 \\
\hline 3. Friend & 76 & $(26.7)$ & 37 & (27.4) & 39 & $(26.0)$ & n.s. \\
\hline 4. Teacher & 14 & $(4.9)$ & 12 & $(8.9)$ & 2 & (1.3) & .004 \\
\hline 5. Other & 13 & $(4.6)$ & 7 & $(5.2)$ & 6 & $(4.0)$ & n.s. \\
\hline$\dagger 6$. Medical professional & 4 & (1.4) & 3 & $(0.2)$ & 1 & $(0.7)$ & n.s. \\
\hline \multicolumn{8}{|c|}{ Satisfaction with sexual Knowledge } \\
\hline Yes & 29 & $(10.2)$ & 9 & $(6.7)$ & 20 & (13.3) & \multirow{2}{*}{ n.s. } \\
\hline No & 256 & $(89.8)$ & 126 & (93.3) & 130 & (86.7) & \\
\hline
\end{tabular}

Chi-square test, note " $\dagger$ " Fisher's exact test

Table 3. Sex education and education on STI prevention by gender

\begin{tabular}{|c|c|c|c|c|c|c|c|c|}
\hline & & \multicolumn{2}{|c|}{$\begin{array}{c}\text { Total } \\
(\mathrm{N}=285)\end{array}$} & \multicolumn{2}{|c|}{$\begin{array}{c}\text { Male } \\
(\mathrm{n}=135)\end{array}$} & \multicolumn{2}{|c|}{$\begin{array}{l}\text { Fe male } \\
(\mathrm{n}=150)\end{array}$} & \multirow[b]{2}{*}{$\mathrm{p}$} \\
\hline & & & (\%) & $\mathrm{n}$ & $(\%)$ & $\mathrm{n}$ & $(\%)$ & \\
\hline \multirow{3}{*}{$\begin{array}{l}\text { Sex education experience } \\
\text { (multi_response) }\end{array}$} & Ele mentary school & 248 & $(87.0)$ & 116 & $(85.9)$ & 132 & $(88.0)$ & n.s. \\
\hline & Junior high school & 263 & $(92.3)$ & 119 & $(88.1)$ & 144 & $(96.0)$ & .015 \\
\hline & High school & 253 & $(88.8)$ & 114 & $(84.4)$ & 139 & $(92.7)$ & .038 \\
\hline \multirow[t]{4}{*}{ Main sex educator } & Teachers & 214 & $(75.1)$ & 104 & $(77.0)$ & 110 & $(73.3)$ & n.s. \\
\hline & Frie nds & 28 & $(9.8)$ & 16 & (11.9) & 12 & $(8.0)$ & n.s. \\
\hline & Other & 22 & $(7.7)$ & 7 & $(5.2)$ & 15 & $(10.0)$ & n.s. \\
\hline & Parents & 21 & $(7.4)$ & 8 & $(5.9)$ & 13 & $(8.7)$ & n.s. \\
\hline \multirow{9}{*}{$\begin{array}{l}\text { Sex education } \\
\text { (multi_response) }\end{array}$} & Fertilization & 222 & $(78.2)$ & 97 & $(71.9)$ & 125 & $(83.3)$ & .022 \\
\hline & Pregnancy & 204 & $(71.8)$ & 93 & $(68.9)$ & 111 & $(74.0)$ & n.s. \\
\hline & Safer sex & 203 & $(71.5)$ & 99 & $(73.3)$ & 104 & $(69.3)$ & n.s. \\
\hline & Menstruation & 184 & $(64.8)$ & 72 & $(53.3)$ & 112 & $(74.7)$ & .000 \\
\hline & Healthy life & 180 & $(63.4)$ & 88 & $(65.2)$ & 92 & $(61.3)$ & n.s. \\
\hline & $\begin{array}{l}\text { Reproductive mechanism of } \\
\text { boys/girls }\end{array}$ & 160 & $(56.3)$ & 82 & $(60.7)$ & 78 & $(52.0)$ & n.s. \\
\hline & Sexual re la tionship & 128 & $(45.1)$ & 73 & $(54.1)$ & 55 & $(36.7)$ & .003 \\
\hline & Premarital sex & 80 & $(28.2)$ & 50 & $(37.0)$ & 30 & $(20.0)$ & .002 \\
\hline & Family planning & 50 & $(17.6)$ & 27 & $(20.0)$ & 23 & $(15.3)$ & n.s. \\
\hline \multirow[t]{3}{*}{ Education on STI prevention } & Prevention of STI & 169 & $(59.5)$ & 84 & $(62.2)$ & 85 & $(56.7)$ & n.s. \\
\hline & Symptoms of STI & 134 & $(47.2)$ & 64 & $(47.4)$ & 70 & $(46.7)$ & n.s. \\
\hline & Treatment of STI & 92 & $(32.4)$ & 61 & $(45.2)$ & 31 & $(20.7)$ & .000 \\
\hline
\end{tabular}

Chi'square test 
Students received sexual knowledge at school and from several sex educators (Table 3 ). The results showed that 29 $(10.2 \%)$ students were satisfied with the sexual knowledge, and there was no significant difference among gender $(\mathrm{p}=$ $0.06)$.

\subsection{Sex Education and Education on STI Prevention by Gender}

Around $90 \%$ of students received sex education from elementary school to high school. Females received more sex education than males during junior high school and high school $(\mathrm{p}=.015$ and $\mathrm{p}=.038)$. The main sex educator from whom students learned sexual knowledge was a teacher for $75 \%$ of students and friends for $9.8 \%$. Contents of sex education were mainly "fertilization" (78.2\%), "pregnancy" (71.8\%), and "safer sex" (71.5\%). Regarding education on STIs, $59.5 \%$ was on prevention, $47.2 \%$ on symptoms, and $32.4 \%$ on treatment (Table 3 ). Regarding "treatment of STIs," males received more education than females and there was a significant difference among gender $(\mathrm{p}=.000)$.

\subsection{Knowledge on STI Prevention}

The STI knowledge test was used to test knowledge on STI prevention. The results are shown in Table 4. The mean scores were $9.78 \pm 1.45$ in total, $9.73 \pm 1.49$ for males and $9.83 \pm 1.43$ for females, showing no significant difference among gender. More than $90 \%$ of participants selected the correct answer to "AIDS is a type of STI," "STIs are one of the causes of abortion," "You need to have a medical examination if you have symptoms such as itching, pain, and odor from your sex organs," and "Sexually transmitted infections (STIs) are transmitted by sexual intercourse." On the other hand, $40.4 \%$ selected the correct answer to "STI symptoms in females are stronger than in males" and $58.9 \%$ selected the correct answer to "Some STIs are asymptomatic." Regarding the $68.8 \%$ who selected "STIs are caused only by viruses," more females selected the correct answer than males, showing a significant difference among gender $(\mathrm{p}=.003)$.

Table 4. Comparison of knowledge test on STIs by gender

\begin{tabular}{|c|c|c|c|c|c|c|c|c|c|c|}
\hline & & \multicolumn{4}{|c|}{$\begin{array}{c}\text { Total } \\
(\mathrm{N}=285)\end{array}$} & \multirow{2}{*}{\multicolumn{2}{|c|}{$\begin{array}{c}\begin{array}{c}\text { Male } \\
(\mathrm{n}=135)\end{array} \\
\text { TRUE }\end{array}$}} & \multirow{2}{*}{\multicolumn{2}{|c|}{$\begin{array}{c}\begin{array}{c}\text { Female } \\
(\mathrm{n}=150)\end{array} \\
\text { TRUE }\end{array}$}} & \multirow[b]{3}{*}{$\mathrm{p}$} \\
\hline & & \multicolumn{2}{|c|}{ TRUE } & \multicolumn{2}{|c|}{ FALSE } & & & & & \\
\hline & & $\mathrm{n}$ & $(\%)$ & $\mathrm{n}$ & $(\%)$ & $\mathrm{n}$ & $(\%)$ & $\mathrm{n}$ & $(\%)$ & \\
\hline \multicolumn{11}{|c|}{ Knowledge test on STIs } \\
\hline 1) & $\begin{array}{l}\text { Sexual transmitted infections (STIs) are transmitted by se xual } \\
\text { in tercourse.(Yes) }\end{array}$ & 239 & $(83.9)$ & 46 & $(16.1)$ & 113 & $(83.7)$ & 126 & $(84.0)$ & n.s. \\
\hline 2) & STI are cause d only by viruse s.(No) & 196 & $(68.8)$ & 89 & $(31.2)$ & 81 & $(60.3)$ & 115 & $(76.7)$ & .003 \\
\hline 3) & Some STIs are asy mptomatic.(Yes) & 168 & $(58.9)$ & 117 & $(41.1)$ & 77 & $(57.0)$ & 91 & $(60.7)$ & n.s. \\
\hline 4) & STI symptoms in females are stronger than those in males.(No) & 115 & $(40.4)$ & 170 & $(59.6)$ & 56 & $(41.5)$ & 59 & $(39.3)$ & n.s. \\
\hline 5) & STIs are one of the cause s of abortion.(Yes) & 260 & $(91.2)$ & 25 & $(8.8)$ & 124 & (91.9) & 136 & $(90.7)$ & n.s. \\
\hline 6) & Some STIs can be passed from mother to fetus.(Yes) & 254 & $(89.1)$ & 31 & $(10.9)$ & 122 & $(90.4)$ & 132 & $(88.0)$ & n.s. \\
\hline 7) & $\begin{array}{l}\text { Some STIs can spread through mucous membranes of any } \\
\text { organs.(Yes) }\end{array}$ & 234 & $(82.1)$ & 51 & $(17.9)$ & 113 & $(83.7)$ & 121 & $(80.7)$ & n.s. \\
\hline 8$) \dagger$ & AIDS is a type of STI.(Yes) & 271 & $(95.1)$ & 14 & $(4.9)$ & 131 & $(97.0)$ & 140 & $(93.3)$ & n.s. \\
\hline 9) & $\begin{array}{l}\text { Human papillo mavirus (HPV) is the cause of cervical } \\
\text { cancer.(Yes) }\end{array}$ & 225 & $(78.9)$ & 60 & (21.1) & 107 & $(79.3)$ & 118 & $(78.7)$ & n.s. \\
\hline $\begin{array}{l}\text { 10) } \\
\dagger\end{array}$ & $\begin{array}{l}\text { It is important to maintain a he althy lifestyle (good nutrition, } \\
\text { good sleep habits, etc.) for the prevention of cervical }\end{array}$ & 273 & $(95.8)$ & 12 & $(4.2)$ & 131 & $(97.0)$ & 142 & $(94.7)$ & n.s. \\
\hline 11) $\dagger$ & $\begin{array}{l}\text { Smoking or second-hand smoking is a risk for cancer in } \\
\text { general(Yes) }\end{array}$ & 279 & $(97.9)$ & 6 & $(2.1)$ & 131 & $(97.0)$ & 148 & $(98.7)$ & n.s. \\
\hline 12) $\dagger$ & $\begin{array}{l}\text { You need to have a medical examination if you have symptoms } \\
\text { such as itch, pain, and odor from your sex organs.(Yes) }\end{array}$ & 274 & $(96.1)$ & 11 & $(3.9)$ & 128 & $(94.8)$ & 146 & $(97.3)$ & n.s. \\
\hline & Mean $\pm \mathrm{SD}$ of total score (Max score $=12)$ & \multicolumn{4}{|c|}{$9.78 \pm 1.45$} & \multicolumn{2}{|c|}{$9.73 \pm 1.49$} & \multicolumn{2}{|c|}{$9.83 \pm 1.43$} & n.s. \\
\hline
\end{tabular}




\subsection{Sexual Abstinence Behavior}

The Sexual Abstinence Behavior Scale (SABS) is shown in Table 5. Regarding "Decision-making (Tell yourself you were making the right decision by waiting to have sex)," $32.6 \%$ of males and $28.7 \%$ of females selected "Somewhat disagree." Moreover, $23.0 \%$ of males selected "Strongly disagree." Second, regarding "Interacting (Say No to sex)," $46.7 \%$ of males and $72.7 \%$ of females selected "Strongly agree." The majority of students selected "Agree." Third, regarding "Negotiation (Tell her/him that you want to wait to have sex)," $40.0 \%$ of males and $64 \%$ of females selected
"Strongly agree." Lastly, regarding "Peer Pressure (Avoid being pressured into having sex by making sure you are out with a group of people)," $25.2 \%$ of males and $52.7 \%$ of females selected "Strongly agree." These results show that female students practiced sexual abstinence behavior more than males, with a significant difference among gender ( $p$ $=.000)$. The ranking of a large proportion among the four items were "Interacting" $(46.7 \%$ of males and $72.7 \%$ of females), "Negotiation" (40.0\% and 64\%), "Peer Pressure" $(25.2 \%$ and $52.7 \%)$ and "Decision-making" (14.8\% and $20.0 \%)$.

Table 5. Comparison of Sexual Abstinence Behavior by gender

\begin{tabular}{|c|c|c|c|c|c|c|c|}
\hline & $\begin{array}{c}6=\text { Strong } \\
\text { agree }\end{array}$ & $5=$ Agree & $\begin{array}{c}4=\text { Somewhat } \\
\text { agree }\end{array}$ & $\begin{array}{c}3=\text { Some what } \\
\text { disagree }\end{array}$ & $2=$ Disagree & $\begin{array}{c}1=\text { Strong } \\
\text { disagree }\end{array}$ & $\mathrm{p}$ \\
\hline 1) Decision-making & $\mathrm{n}=50$ & $\mathrm{n}=38$ & $\mathrm{n}=60$ & $\mathrm{n}=87$ & $\mathrm{n}=16$ & $\mathrm{n}=34$ & \multirow[b]{2}{*}{.000} \\
\hline $\begin{array}{l}\text { Male }(n=135) \\
\text { Female }(n=150)\end{array}$ & $\begin{array}{l}20(14.8 \%) \\
30(20.0 \%)\end{array}$ & $\begin{array}{c}11(8.1 \%) \\
27(18.0 \%)\end{array}$ & $\begin{array}{l}22(16.3 \%) \\
38(25.3 \%)\end{array}$ & $\begin{array}{l}44(32.6 \%) \\
43(28.7 \%)\end{array}$ & $\begin{array}{l}7(5.2 \%) \\
9(6.0 \%)\end{array}$ & $\begin{array}{c}31(23.0 \%) \\
3(2.0 \%)\end{array}$ & \\
\hline 2) Interacting & $\mathrm{n}=172$ & $\mathrm{n}=56$ & $\mathrm{n}=26$ & $\mathrm{n}=21$ & $\mathrm{n}=1$ & $\mathrm{n}=9$ & \multirow[b]{2}{*}{.000} \\
\hline $\begin{array}{l}\text { Male } \quad(\mathrm{n}=135) \\
\text { Female }(\mathrm{n}=150)\end{array}$ & $\begin{array}{r}63(46.7 \%) \\
109(72.7 \%)\end{array}$ & $\begin{array}{l}32(23.7 \%) \\
24(16.0 \%)\end{array}$ & $\begin{array}{c}17(12.6 \%) \\
9(6.0 \%)\end{array}$ & $\begin{array}{l}13(9.6 \%) \\
8(5.3 \%)\end{array}$ & $\begin{array}{l}1(0.7 \%) \\
0(0.0 \%)\end{array}$ & $\begin{array}{l}9(6.7 \%) \\
0(0.0 \%)\end{array}$ & \\
\hline 3) Negotiation & $\mathrm{n}=150$ & $\mathrm{n}=62$ & $\mathrm{n}=33$ & $\mathrm{n}=24$ & $\mathrm{n}=5$ & $\mathrm{n}=11$ & \multirow[b]{2}{*}{.000} \\
\hline $\begin{array}{l}\text { Male } \quad(n=135) \\
\text { Female }(n=150)\end{array}$ & $\begin{array}{l}54(40.0 \%) \\
96(64.0 \%)\end{array}$ & $\begin{array}{l}27(20.0 \%) \\
35(23.3 \%)\end{array}$ & $\begin{array}{c}24(17.8 \%) \\
9(6.0 \%)\end{array}$ & $\begin{array}{c}17(12.6 \%) \\
7(4.7 \%)\end{array}$ & $\begin{array}{l}3(2.2 \%) \\
2(1.3 \%)\end{array}$ & $\begin{array}{c}10(7.4 \%) \\
1(0.7 \%)\end{array}$ & \\
\hline 4) Peer pressure & $\mathrm{n}=113$ & $\mathrm{n}=57$ & $\mathrm{n}=37$ & $\mathrm{n}=40$ & $\mathrm{n}=10$ & $\mathrm{n}=28$ & \multirow{3}{*}{.000} \\
\hline Male $\quad(n=135)$ & $34(25.2 \%)$ & $24(17.8 \%)$ & $18(13.3 \%)$ & $27(20.0 \%)$ & $8(5.9 \%)$ & $24(17.8 \%)$ & \\
\hline Female $(n=150)$ & $79(52.7 \%)$ & $33(22.0 \%)$ & $19(12.7 \%)$ & $13(8.7 \%)$ & $2(1.3 \%)$ & $4(2.7 \%)$ & \\
\hline
\end{tabular}

Table 6. Concern about STIs and coping behavior for STI by Gender

\begin{tabular}{|c|c|c|c|c|c|c|c|c|}
\hline & & \multicolumn{2}{|c|}{$\begin{array}{c}\text { Total } \\
(\mathrm{N}=285)\end{array}$} & \multicolumn{2}{|c|}{$\begin{array}{c}\text { Male } \\
(\mathrm{n}=135)\end{array}$} & \multicolumn{2}{|c|}{$\begin{array}{l}\text { Female } \\
(\mathrm{n}=150)\end{array}$} & \multirow[b]{2}{*}{$\mathrm{p}$} \\
\hline & & $\mathrm{n}$ & $(\%)$ & $\mathrm{n}$ & $(\%)$ & $\mathrm{n}$ & $(\%)$ & \\
\hline \multicolumn{9}{|c|}{ Concern about STIs } \\
\hline \multirow{2}{*}{\multicolumn{2}{|c|}{ Do you have worried about STI }} & 39 & $(14.0)$ & 18 & (13.8) & 21 & $(14.2)$ & \multirow{2}{*}{ n.s. } \\
\hline & & 239 & $(86.0)$ & 112 & $(86.2)$ & 127 & $(85.8)$ & \\
\hline \multicolumn{9}{|c|}{ Medical check-up } \\
\hline \multirow[t]{2}{*}{ 1. Clamydia } & Yes & 241 & $(84.6)$ & 111 & $(82.2)$ & 130 & $(86.7)$ & \multirow{2}{*}{ n.s. } \\
\hline & No & 44 & $(15.4)$ & 24 & (17.8) & 20 & (13.3) & \\
\hline \multicolumn{9}{|c|}{ Reason "No" } \\
\hline \multicolumn{2}{|c|}{ 1) Symptoms will reduce } & 26 & $(60.5)$ & 11 & $(47.8)$ & 15 & $(75.0)$ & n.s. \\
\hline \multicolumn{2}{|c|}{$\dagger$ 2) Ashamed of examination } & 15 & $(34.9)$ & 2 & $(8.7)$ & 13 & $(65.0)$ & .000 \\
\hline \multicolumn{2}{|c|}{$\dagger 3$ ) Afraid for parents } & 9 & $(20.9)$ & 3 & $(13.0)$ & 6 & $(30.0)$ & n.s. \\
\hline \multicolumn{2}{|c|}{$\dagger$ 3) No needed Medical Examinatj } & 9 & $(20.9)$ & 8 & $(34.8)$ & 1 & $(5.0)$ & .024 \\
\hline \multirow[t]{2}{*}{ 2. Gonorrhea } & Yes & 264 & $(92.6)$ & 123 & $(91.1)$ & 141 & $(94.0)$ & \multirow{2}{*}{ n.s. } \\
\hline & No & 21 & (7.4) & 12 & (8.9) & 9 & $(6.0)$ & \\
\hline \multicolumn{9}{|c|}{ Reason "No" } \\
\hline \multicolumn{2}{|c|}{$\dagger$ 1) Symptoms will reduce } & 10 & $(47.6)$ & 4 & $(33.3)$ & 6 & $(66.7)$ & n.s. \\
\hline \multicolumn{2}{|c|}{$\dagger 2$ ) Ashamed of examination } & 6 & $(28.6)$ & 1 & $(8.3)$ & 5 & $(55.6)$ & .046 \\
\hline \multicolumn{2}{|c|}{$\dagger 3)$ Afraid for parents } & 6 & $(28.6)$ & 3 & $(25.0)$ & 3 & (33.3) & n.s. \\
\hline \multicolumn{2}{|c|}{$\dagger$ 4) No needed Medical Examinatj } & 3 & $(14.3)$ & 3 & $(25.0)$ & 0 & 0.0 & n.s. \\
\hline
\end{tabular}

Chi'squire test, note " $\dagger$ " Fishr's Exact- test 


\subsection{Concerns about STIs and Coping Behavior for STIs}

Concerns about STIs and coping behavior for STIs are shown in Table 6. Regarding STIs, $14 \%$ of students were worried about STIs. There was no significant difference among gender. Regarding coping behavior for STIs, $84.6 \%$ of students answered that they would go for a medical checkup if chlamydia symptoms were present, and $92.6 \%$ of students answered they would do so for gonorrhea symptoms. There was no significant difference among gender $(\mathrm{p}=0.30)$.

Among those students who would not go for a medical checkup, their reasons were as follows. For chlamydia, $60.5 \%$ of students selected "The symptoms will reduce," $34.9 \%$ selected "Shame of examination," and 20.9\% selected "Afraid of parents" or "Don't need a medical examination." For gonorrhea, 47.6\% of students selected "The symptoms will reduce," 28.6\% selected "Shame of examination" or "Afraid of parents," and 14.3\% selected "Don't need a medical examination." "Shame of examination" and "Afraid of parents" were answered by more female students than male students, and there was a significant difference among gender $(p=.000)$

\subsection{Comparison between Education on STI Prevention, etc. on Satisfaction with Sexual Knowledge, STI Knowledge Test, and Medical Checkup}

Table 7 compares education on STI prevention, etc. on satisfaction with sexual knowledge, STI knowledge test and medical checkup. Comparing students who received education on STI prevention, etc., there was no significant difference regarding satisfaction with sexual knowledge, STI knowledge test, SABS and medical checkup for chlamydia symptoms (p $=0.633, p=0.148, p=0.957$ and $p=0.485)$. However, there were significant differences for gonorrhea $(p=.003)$. Second, comparing students who received education on STI symptoms, etc., there was a significant difference in the STI knowledge test $(p=.035)$. There were no differences in satisfaction with sexual knowledge $(p=0.803)$, SABS $(p=0.542)$ or medical checkup ( $p=0.377$ for chlamydia, $p=0.691$ for gonorrhea). Lastly, comparing students who received education on STI treatment, etc., there were significant differences in the STI knowledge test and SABS $(p=.011, p=.033)$. However, there were no differences in satisfaction with sexual knowledge $(p=0.068)$ or medical checkup $(p=0.943$ for chlamydia, $\mathrm{p}=0.089$ for gonorrhea).

Table 7. Comparison between education on STI prevention, etc. on satisfaction with sexual knowledge, STI knowledge test, and medical checkup, N=285

\begin{tabular}{|c|c|c|c|c|c|c|c|c|c|c|c|c|c|c|}
\hline & & \multicolumn{3}{|c|}{$\begin{array}{l}\text { Satisfaction with sexual } \\
\text { knowledge }\end{array}$} & \multirow{2}{*}{$\begin{array}{l}\text { STI Knowledge test } \\
\text { (Max score }=12) \\
\text { Mean } \pm \text { SD }\end{array}$} & \multirow{2}{*}{\multicolumn{2}{|c|}{$\begin{array}{l}\text { Sexual Abstinence Behavior Score } \\
(\text { Max score }=24) \\
\text { Mean } \pm \text { SD }\end{array}$}} & \multirow[b]{2}{*}{$\mathrm{p}$} & \multicolumn{2}{|c|}{$\begin{array}{l}\text { Medical checkup of } \\
\text { Chlamydia }\end{array}$} & \multicolumn{4}{|c|}{$\begin{array}{l}\text { Medical checkup of } \\
\text { Gonorrhea }\end{array}$} \\
\hline & & Yes & NO & $\mathrm{p}$ & & & & & Yes & $\mathrm{NO}$ & $\mathrm{p}$ & Yes & NO & $\mathrm{p}$ \\
\hline \multirow[t]{2}{*}{ Prevention of STI } & $\begin{array}{c}\text { Yes } \\
(\mathrm{n}=169)\end{array}$ & $16(9.5 \%)$ & $153(90.5 \%)$ & \multirow[b]{2}{*}{ n.s. } & $9.92 \pm 1.25$ & \multirow[b]{2}{*}{ n.s. } & $18.40 \pm 4.60$ & \multirow[b]{2}{*}{ n.s. } & $145(85.8 \%)$ & $24(14.2 \%)$ & \multirow[b]{2}{*}{ n.s. } & $163(96.4 \%)$ & $6(3.6 \%)$ & \multirow[b]{2}{*}{.003} \\
\hline & $\begin{array}{c}\text { No } \\
(\mathrm{n}=116)\end{array}$ & $13(11.2 \%)$ & $103(88.8 \%)$ & & $9.58 \pm 1.70$ & & $18.54 \pm 4.42$ & & $96(82.8 \%)$ & $20(17.2 \%)$ & & $101(87.1 \%)$ & $15(12.9 \%)$ & \\
\hline \multirow[t]{2}{*}{ Symptoms of STI } & $\begin{array}{c}\text { Yes } \\
(\mathrm{n}=134)\end{array}$ & $13(9.7 \%)$ & $121(90.3 \%)$ & \multirow{2}{*}{ n.s. } & $9.93 \pm 1.51$ & \multirow{2}{*}{.035} & $18.39 \pm 4.97$ & \multirow{2}{*}{ n.s. } & $116(86.6 \%)$ & $18(13.4 \%)$ & \multirow{2}{*}{ n.s. } & $125(93.3 \%)$ & $9(6.7 \%)$ & \multirow{2}{*}{ n.s. } \\
\hline & $\begin{array}{c}\text { No } \\
(\mathrm{n}=151)\end{array}$ & $16(10.6 \%)$ & $135(89.4 \%)$ & & $9.65 \pm 1.40$ & & $18.52 \pm 4.09$ & & $125(82.8 \%)$ & $26(17.2 \%)$ & & $139(92.1 \%)$ & $12(7.9 \%)$ & \\
\hline \multirow[t]{2}{*}{ Treatment of STI } & $\begin{array}{c}\text { Yes } \\
(\mathrm{n}=92)\end{array}$ & $5(5.4 \%)$ & $87(94.6 \%)$ & \multirow[b]{2}{*}{ n.s. } & $10.10 \pm 1.20$ & \multirow[b]{2}{*}{.011} & $17.33 \pm 5.37$ & \multirow[b]{2}{*}{.033} & $78(84.8 \%)$ & $14(15.2 \%)$ & \multirow[b]{2}{*}{ n.s. } & $89(96.7 \%)$ & $3(3.3 \%)$ & \multirow[b]{2}{*}{ n.s. } \\
\hline & $\begin{array}{c}\text { No } \\
(\mathrm{n}=193)\end{array}$ & $24(12.4 \%)$ & $169(87.6 \%)$ & & $9.63 \pm 1.55$ & & $19.00 \pm 3.96$ & & $163(84.5 \%)$ & $30(15.5 \%)$ & & $175(90.7 \%)$ & $18(9.3 \%)$ & \\
\hline
\end{tabular}

Chi-squre test and Mann-Whitney U test 


\section{Discussion}

This is the first study to demonstrate that high school students in Korea receive insufficient education on the prevention of STIs, which may result in students' poor understanding of the symptoms of STIs and the need to receive a medical checkup for STIs.

Our results showed that regarding knowledge on STI prevention among Korean students, 32.4-59.2\% of students had received education on STI prevention. Furthermore, $96.1 \%$ of students selected "You need to have a medical examination if you have symptoms such as itching, pain, and odor from your sex organs." However, only around $60 \%$ of students recognized asymptomatic STIs and $40 \%$ of students thought that "STI symptoms in females are stronger than in males." Students who received education on symptoms of STIs obtained significantly higher scores in the knowledge test than others $(p=.035)$. As the background, $74.1 \%$ of students got information on sex from school, $57.4 \%$ from the Internet and $40.4 \%$ from friends. Moreover, $32.3 \%$ of students selected parents as the best counselor about sexual problems, but $30 \%$ of male students had no one to consult with, and so they did not understand the prevention of STIs and only $10.2 \%$ were satisfied with their sexual knowledge. However, the score for sexual knowledge in this study was higher than that of previous studies when calculated as a percentage [20, 21]. This shows that the sex education rate found in this study was high, implying that sex education at school had some influence on the students' sexual knowledge. Among the survey questions, "AIDS is a type of STI" and "STIs are one of the causes of abortion" showed very high correct response rates. However, the percentages of students giving correct answers regarding STI symptoms occurring more strongly in females than in males and some STIs not having symptoms were lower; these results are consistent with findings that HIV knowledge was high but knowledge about other STIs was low $[22,23]$. This can be explained by the fact that an HIV awareness campaign was carried out worldwide [22], while students at schools do not receive sufficient education on STI prevention.

Secondly, regarding Sexual Abstinence Behavior (SAB) for students, their scores were high: Interacting $5.23 \pm 1.22$, Negotiation 5.04 \pm 1.33 , Peer Pressure 4.49 \pm 1.65 , and Decision-making $3.71 \pm 1.54$. Such behavior may be why the majority of students did not have experience of sexual intercourse. Therefore, $14 \%$ of students were worried about STIs. However, SAB had no relation with education on STI prevention. Regarding coping behavior for STIs, $84.6 \%$ of students said they would go for a checkup for chlamydia, and $92.6 \%$ would do so for gonorrhea. However, 7.4-15.4\% of students did not do so because "The symptoms will reduce," "Shame of examination," "Afraid of parents" and "Don't need a medical examination." Both of them showed no significant difference among gender. These results suggest that Korean high school students are close to their parents and consult them about sexual problems. Regarding coping behavior for STIs, female students may not go for a checkup because they feel shame. Incidentally, students who received education on STI prevention selected that they would go for a checkup more than others $(p<0.01)$. On the other hand, there were no significant differences among students who received education on STIs or treatment of STIs. Therefore, there was no relation between education on STI prevention and the STI knowledge test, and this study found that students were not satisfied with their sexual knowledge. High school students in Korea seem to need appropriate education on STI prevention. The same applies to Thai high school students regarding STI education [17], too. On the other hand, Thai students consult their parents and receive information on STIs. However, in Korea, the rate of students who chose parents as counselors and information sources was low. This may be true not only for adolescents but also due to the influence of Korean culture. Therefore, sex educators need to provide knowledge on sexuality along with the development stage and consider students' individual sexual issues, as was found in the Thai study [17]. There may be a need for health professionals to provide training to teachers in schools on how to teach STI prevention [24]. As was found in a previous study, many students said they would not receive a checkup because of the need for an internal examination; the previous study found that unmarried women felt very uncomfortable visiting obstetricians and gynecologists, and were very reluctant to tell a male doctor about their experience of sexual intercourse or to expose their genital organs [25]. This is due to the negative social perceptions of young or unmarried women visiting obstetricians and gynecologists. However, obstetricians and gynecologists say that the first visit to them should be made at age 13-15 if possible, so that adolescent girls can receive healthcare information and preventive healthcare services, and so that diseases may be discovered early [25]. It is considered necessary to educate both students and parents on this information to reduce the embarrassment of visiting obstetricians and gynecologists.

The limitations of this study are as follows. Since the topic of STIs can be sensitive and uncomfortable for adolescents, participants are highly likely to have responded to questions insincerely. However, this study is significant because it examined the status of STI prevention, along with related knowledge of Korean students and how they would cope with STI symptoms. Therefore, this study provides basic data for an educational program to prevent STIs among adolescents and to enable them to appropriately cope with STIs when they are contracted.

\section{Conclusions}

This study examined Korean adolescents' knowledge on STI prevention and how they cope with STI symptoms. 
The results showed that they did not receive appropriate education on STI prevention, they felt they had insufficient sexual knowledge, and they did not understand the symptoms of STIs. Due to lack of knowledge and shame, they did not receive checkups as coping behavior. However, one out of three students consulted with their parents or friends about sexual problems. Therefore, sex education including STIs, prevention, and coping behavior needs to be provided at high schools before students encounter sexual events. It may also be necessary to set up health care clinics where adolescents can receive checkups and advice.

\section{Acknowledgements}

We wish to thank the participants, public high schools, and teachers in Korea who assisted this study.

\section{REFERENCES}

[1] World Health Organization (WHO), Sexually transmitted infection (STIs), Geneva: WHO, 2016, http://who.int/mediacentre/factsheets/fs 110/en/

[2] Satterwhite CL et al., Sexually transmitted infections among US women and men: prevalence and incidence estimates, 2008, Sexually Transmitted Diseases, 2013, 40(3):187-193.

[3] Forhan SE et al., Prevalence of sexually transmitted infections among female adolescents aged 14 to 19 in the United States, Pediatrics, 2009, 124(6):1505-1512. http://dx.doi.org/10.1542/peds.2009-0674

[4] Lee SY et al., Sexually transmitted infections and first sexual intercourse age in adolescents: The nationwide retrospective cross-sectional study, The Journal of Sexual Medicine, 2015, 12(12):2313-2323.

[5] Health Insurance Review \& Assessment Service, Public Interest Disease Statistics, Wonju: Health Insurance Review \& Assessment Service, 2013, http:/opendata.hira.or.kr/op/opc/olapMfrnIntrsIlnsInfo.do \#none

[6] Korea Centers for Disease Control and Prevention (KCDC), The twelfth Korea Youth Risk Behavior Web Based Survey, 2016, Cheongju: KCDC, 2016, http://www.cdc.go.kr/CDC/contents/CdcKrContentView.js p? cid=77749\&menuIds=HOME001-MNU1130-MNU239 3-MNU2749

[7] Gewirtzman A et al., Sexually transmitted infections and sexually transmitted diseases, In: Gewirtzman A et al., eds., Epidemiology of Sexually Transmitted Infections, Berlin Heidelberg, Germany: Springer, 2011, pp. 13-34. https://dx.doi.org/10.1007/978-3-642-14663-3_2

[8] Lee J et al., The evaluation and risk assessment of sexually transmitted disease in Korean adolescents at risk,International Journal of Infectious Diseases, 2016, 45:202. http://dx.doi.org/10.1016/j.ijid.2016.02.465
[9] WHO, Sexually transmitted infections among adolescents: the need for adequate health services, Geneva: WHO, 2016.

[10] Trettin B, Vestergaard T and Stensgaard A, Understanding young people's barriers to sexually transmitted disease screening and meeting their needs: A focus group study, Journal of Nursing Education and Practice, 2015, 5(6):81. https://dx.doi.org/10.5430/jnep.v5n6p81

[11] Tilson EC et al., Barriers to asymptomatic screening and other STD services for adolescents and young adults: focus group discussions, BMC Public Health, 2004, 4(1): 21 https://dx.doi.org/10.1186/1471-2458-4-21

[12] Newby KV, Wallace LM and French DP, How do young adults perceive the risk of chlamydia infection? A qualitative study, British Journal of Health Psychology, 2012, 17(1):144-154.

https://dx.doi.org/10.1111/j.2044-8287.2011.02027.x

[13] Kim EJ and Ha SM, A study on sexual consciousness, the actual condition of school sex education and its need in high school students. Journal of Child Welfare and Development, 2016, 14(2):19-39.

[14] Lee HS et al., A study on the level of sex education, sexual knowledge, sexual attitude and sexual allowance among high school students. Education Culture Research, 2015, 21(3):231-252.

[15] Kim SH and Lee CY. Factors affecting sexually transmitted infections in South Korean high school students, Public Health Nursing, 2016, $33 \quad$ (3): 179-188. https://dx.doi.org/10.1111/phn.12211

[16] Gwon SH and Lee CY. Factors influencing sexually transmitted infections among adolescents in South Korea, International Nursing Review, 2016, 63 (1): 68-77. http://dx.doi.org/ 10.1111/inr.12206

[17] Yamaguchi $\mathrm{Y}$ et al., Knowledge, attitude, and practice concerning the prevention of STIs among high school students in Northern Thailand, Universal Journal of Public Health, 2016, 4 (1): 8-15. https://dx.doi.org/10.13189/ujph.2016.040102

[18] Beaton DE et al., Guidelines for the process of cross-cultural adaptation of self-report measures, Spine, 2000, 25(24):3186-3891.

[19] Anne E.Norris, Leslie F.Clark, Sondra Magnus, Sexual abstinence and the Sexual Abstinence Behavior Scale, Journal of Pediatric Health Care, May-June 2003:17(3)140-144

[20] Jones NR and Haynes R, The association between young people's knowledge of sexually transmitted diseases and their behaviour: A mixed methods study Health, Risk \& Society, 2006, 8 (3): 293-303. http://dx.doi.org/10.1080/13698570600871851

[21] Wolfers M, de Zwart O and Kok G, Adolescents in The Netherlands underestimate risk for sexually transmitted infections and deny the need for sexually transmitted infection testing, AIDS Patient Care STDS, 2011, 25(5):311-319. https://dx.doi.org/10.1089/apc.2010.0186

[22] Samkange-Zeeb F, Mikolajczyk RT and Zeeb H, Awareness and knowledge of sexually transmitted diseases among secondary school students in two German cities, 
Journal of Community Health, 2013, 38(2):293-300. https://dx.doi.org/10.1007/s10900-012-9614-4

[23] Samkange-Zeeb FN, Spallek L and Zeeb H, Awareness and knowledge of sexually transmitted diseases (STDs) among school-going adolescents in Europe: a systematic review of published literature, BMC Public Health, 2011, 11(1):727. https://dx.doi.org/10.1186/1471-2458-11-727

[24] Barr EM1, Goldfarb ES, Russell S, Seabert D, Wallen M,
Wilson KL., Improving sexuality education: the development of teacher-preparation standards, J Sch Health. 2014 Jun; 84(6):396-415.

[25] Lee EJ, A grounded theory analysis on unmarried women's experiences and perceptions of visit to the hospital specialized in obstetrics and gynecology, Korea Science \& Art Forum, 2015, 20:349-364.http://dx.doi.org/10.17548/k saf.2015.06.20.349 